\title{
Contrast enhanced ultrasound for the diagnosis of liver hemangiomas - results of a Romanian multicentre study.
}

\author{
Roxana Şirlii ${ }^{1}$, Ioan Sporea ${ }^{1}$, Daniela Larisa Săndulescu ${ }^{2}$, Alina Popescu ${ }^{1}$, Mirela Dănilă ${ }^{1}$, \\ Adrian Săftoiu ${ }^{2}$, Zeno Spârchez ${ }^{3}$, Radu Badea ${ }^{3}$
}

${ }^{1}$ Department of Gastroenterology and Hepatology, "Victor Babeş" University of Medicine and Pharmacy Timişoara, ${ }^{2}$ Centre for Research in Gastroenterology and Hepatology, University of Medicine and Pharmacy Craiova, ${ }^{3}$ Regional Institute of Gastroenterology and Hepatology "Prof. Dr. Octavian Fodor", "Iuliu Hațieganu" University of Medicine and Pharmacy Cluj Napoca, Romania

\begin{abstract}
Background and aim: Contrast enhanced ultrasound (CEUS) has been proven to be a reliable method for the characterization of focal liver lesions (FLL). The aim of this paper was to evaluate the performance of CEUS for the diagnosis of liver hemangiomas in a large cohort of patients. Material and method: We performed a multicentre prospective study which included successive CEUS examinations from fourteen centers from Romania. CEUS examinations were performed in de novo FLL, using low mechanical index ultrasound, following an intravenous bolus of $2.4 \mathrm{ml}$ SonoVue. CEUS was considered conclusive for hemangioma if a typical pattern was present following contrast (centripetal fill in during the arterial phase, hyperenhanced lesion during venous and late phases). In all cases a reference method was available (contrast CT or MRI or biopsy). The trial was registered in clinicaltrials.gov (Identifier NCT01329458). Results: During February 2011 - May 2015 , 1153 CEUS examinations were performed for the evaluation of de novo FLL. Out of the 1153 de novo FLL, 238 cases were diagnosed as hemangiomas by CEUS (typical enhancing pattern). Contrast CT/MRI and biopsy diagnosed additional 24 hemangiomas. From the 238 cases diagnosed as hemangiomas by CEUS, in 11 the final diagnosis was different. Considering contrast CT/MRI and biopsy as reference methods, CEUS had $90.4 \%$ sensitivity, $98.8 \%$ specificity, $95.4 \%$ positive predictive value, $97.4 \%$ negative predictive value, resulting in $96.9 \%$ diagnostic accuracy for the diagnosis of hemangiomas. Conclusion: CEUS is a sensitive and very specific method for the diagnosis of hemangiomas.
\end{abstract}

Keywords: Contrast Enhanced Ultrasound, focal liver lesions, hemangiomas

\section{Introduction}

Focal liver lesions (FLLs) are frequently found in clinical practice due to the widespread use of ultrasonography (US), either during a routine examination or during the follow-up in chronic hepatitis or in oncologic patients. Of course, a new FLL is a major concern for the patient and for the physician. Standard B mode US is not enough to establish a final diagnosis, excepting cystic lesions (simple cysts and some types of hydatid cysts) and focal fatty lesions [1]. Color and spectral Doppler US

Received 05.09.2015 Accepted 10.10.2015

Med Ultrason

2015, Vol. 17, No 4, 444-450

Corresponding author: Dr. Roxana Şirli

14, Sirius str., ap. 5

300688 Timişoara

Tel: +40-748331232

E-mail: roxanasirli@gmail.com don't add much to the diagnostic of FLL since their ability to detect blood flow at the perfusion level is limited, even if they provide important information regarding the directional blood flow in large vessels. Until a decade ago differential diagnosis among different types of FLLs could be made by contrast enhanced Computed Tomography (CT) or Magnetic Resonance Imaging (MRI), or by biopsy, each one with their advantages, but all sharing the same disadvantage: the time needed to reach a final diagnosis (scheduling issues as well as the time needed to prepare and examine a tissue specimen in biopsies).

Contrast enhanced ultrasonography (CEUS) is a realtime imaging technique, that can be performed immediately after a standard US examination, thus it is able to provide a reliable diagnosis a few minutes after the FLL is discovered. Similar to contrast agents used for CT or MRI, second generation US contrast agents are able 
to show tissue perfusion, and, based on enhancement patterns in arterial, portal, and late vascular phases, to identify different types of FLLs. The value of CEUS in diagnosing FLLs was demonstrated in two national multicentre studies (a German and a French one) [2,3], each including more than 1000 FLL, while CEUS accuracy was proved to be similar to those of contrast enhanced CT and MRI [4,5]. Furthermore, in 2004 the European Federation of Societies for Ultrasound in Medicine and Biology (EFSUMB) issued Guidelines and Recommendations concerning the use of CEUS [6] revised in 2008 [7] and in 2012 [8], the later developed in cooperation with the World Federation for Ultrasound in Medicine and Biology (WFUMB), thus with universal valability.

Hemangioma is the most frequent benign liver tumor, the prevalence varying according to different authors from $1-2 \%$ [9] to $20 \%$ [10], more frequent in women than in men (women:man ratio ranging from $2: 1$ to $5: 1$ ) [11]. They are often solitary, but in $40 \%$ of cases multiple lesions can be found in both liver lobes [12], with diameters ranging from a few millimeters to $15-20 \mathrm{~cm}$, those larger than $5 \mathrm{~cm}$ being known as giant hemangiomas [13].

Hemangiomas are considered to be hamartomas, congenital vascular malformations resulting from the abnormal proliferation of endothelial cells, with incompletely understood etiology, but with certain hormonal influence on tumor growth (hemangiomas enlarge during pregnancy and estro-progestative treatment) $[14,15]$. Other suggested etiologic factors are genetic predisposition [16] as well as an autoimmune mechanism [17].

Microscopically, hemangiomas are composed of cavernous vascular spaces lined by a single layer of flat endothelium, filled with blood, separated by thin fibrous septae. The vascular spaces vary in size and may contain thrombi. In large hemangiomas collagenous scars or fibrous nodules may appear due to thrombosis [18].

Most hemangiomas are asymptomatic, being an incidental finding. Large hemangiomas can cause symptoms such as fullness, discomfort and pain in the right upper quadrant [19]. Acute abdominal pain in a patient with a large hemangioma should be suggestive of thrombosis or bleeding within the tumor and may be accompanied by abnormal liver function tests and fever [20].

In B mode US, hemangiomas typically appear as hyperechoic, well defined lesions, with or without small central regions with decreased echogenicity [21,22]. Another appearance highly suggestive of hemangioma is of a hypo- or isoechoic mass with hyperechoic periphery [23], an aspect encountered also in patients with fatty infiltration. Color Doppler does not improve the accuracy of $\mathrm{B}$ mode US since hemangioma blood flow can be seen by color Doppler only in $10-50 \%$ of cases [24]. The same US aspect can be encountered in some malignant lesions, thus contrast second line imaging methods are needed to confirm the diagnosis, especially in high risk patients, such as cirrhotics or oncologic patients, in which a new FLL is found.

Until a few years ago available second line imaging methods were only contrast enhanced CT or MRI and Technetium-99m pertechnetate-labeled red blood cell pool studies. In recent years, CEUS became a valuable alternative of these techniques. The aim of this paper was to evaluate the performance of CEUS for the diagnosis of liver hemangiomas in a large cohort of patients.

\section{Material and methods}

A multicenter prospective study was conducted during 51 months ( $1^{\text {st }}$ of February $2011-15^{\text {th }}$ of May 2015) in 8 university centers (14 individual departments), and the trial was registered in clinicaltrials.gov (Identifier NCT01329458).

\section{Patients}

The study included consecutive patients (older than 18 years), with one to three newly discovered FLL during B-mode US, regardless of the FLL size. In all patients CEUS was performed and a second line imaging method (contrast CT or MRI) or biopsy were also available, considered to be the reference method. In all patients demographic data, as well as data regarding indication for CEUS, history of chronic hepatopathy or oncologic diseases were recorded.

We excluded from the study: patients who did not agree to participate; patients with contraindication for CEUS (acute myocardial infarction, class III/IV cardiac insufficiency, significant rhythm disorders, pregnant women); patients diagnosed with simple cysts or with hydatid cysts by B-mode ultrasound; patients with known FLL (hepatocellular carcinoma following percutaneous treatment, follow-up of patients with known metastasis or other FLLs, etc); patients in whom second line imaging methods or biopsy were unavailable.

A dedicated website (http://study.umfcv.ro) was developed by the University of Medicine and Pharmacy of Craiova for this study, and the collected data were registered online for each individual patient.

Informed consent for the contrast enhanced study was obtained from every patient. The study protocol was approved by the local Ethical Committee of each center and was in accordance with the Helsinki Declaration of 1975.

\section{Conventional and contrast-enhanced studies}

B-mode US and CEUS were performed in each patient with the same ultrasound machine. Different ma- 
chines were used in different centers, but all had capabilities for low-mechanical index examinations. The amount of contrast agent, as well as the type of ultrasound machine and the operator, were also documented. All contrast studies were performed using dedicated contrast software of each ultrasound machine. CEUS was interpreted by experts from each center, which were blinded to the $\mathrm{CT} / \mathrm{MRI}$ or histology results.

The number, size, location, and ultrasound pattern of FLL were documented after B-mode ultrasound. CEUS was performed with convex probes using a low mechanic index $(0.09-0.11)$ in order to minimize microbubble disruption. Once set, the US scan parameters - such as focal zone and time gain compensation - were not changed throughout the study. One focus was positioned below the lesion.

The contrast agent used was SonoVue ${ }^{\circledR}$ (Bracco SpA, Milan, Italy), a perfluoro gas containing agent, provided as a sterile, lyophilized powder contained in a septumsealed vial. Usually $2.4 \mathrm{~mL}$ of SonoVue ${ }^{\circledR}$ were injected through a peripheral intravenous cannula of sufficient size, followed by a $10-\mathrm{mL}$ saline flush, as per standard protocol $[7,8]$. Lesions enhancement patterns were studied in 3 phases: arterial (10-30 seconds after injection), portal (30-120 seconds), and late phase ( $>120$ seconds) according to EFSUMB recommendations $[7,8]$. The contrast study for each patient lasted 5 minutes after bolus injection and was documented by at least 4 video files no longer than 30 seconds each, containing: B-mode examination, the arterial phase, the portal phase, and the late phase.

The contrast vascular patterns were defined by comparing the FLL's enhancement behavior to the surrounding liver parenchyma and were classified as: homogeneous hyperenhancement - the whole FLL showed global homogeneous contrast enhancement; heterogeneous hyperenhancement - the FLL presented mixed irregular areas of contrast enhancement; rim-like hyperenhancement - a peripheral hyperenhancement limited to $<25 \%$ of the tumor's diameter; isoenhancement - the FLL enhanced similarly to the adjacent parenchyma at the same depth; hypoenhancement - the lesion enhanced less than the adjacent parenchyma at the same depth; wash-out - hypoenhancement in the portal or late phases preceded by hyper or iso-enhancement in the arterial phase.

A CEUS diagnosis of hemangioma was established after the contrast study based on the patterns described in the EFSUMB guidelines [7,8]: centripetal fill-in in the arterial phase, partial/complete centripetal filling and sustained enhancement in portal and late phases (fig 1). A CEUS examination was considered conclusive if, following contrast, the FLL had a typical enhancement

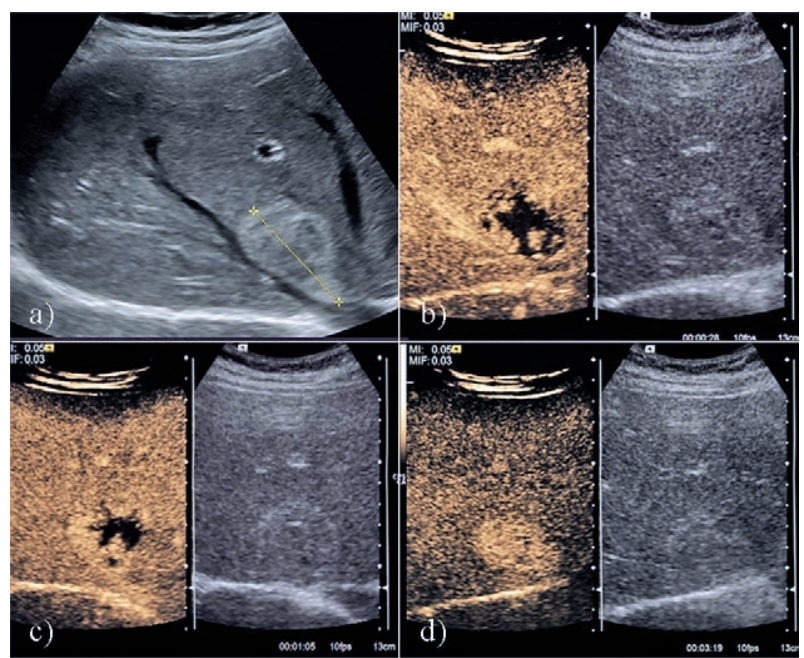

Fig 1. a) Hyperechoic, well delineated inhomogeneous nodule, situated in segment VIII of the liver; b) CEUS - arterial phase: nodular, peripheral enhancement of the nodule can be observed; c) CEUS - portal phase: centripetal peripheral enhancement continues; d) CEUS - late phase: the nodule is completely filled and hyperenhancing.

pattern according to the EFSUMB guidelines [7,8], and inconclusive if the enhancement pattern was not in concordance with these guidelines. The CEUS diagnosis was compared with the final diagnosis which was established based on all available imaging and clinical data: contrast enhanced CT, and/or MRI, and/or histology.

\section{Statistical analysis}

Statistical analysis was performed using the MedCalc program (MedCalc Software, version 12.3.0, Belgium). The accuracy of CEUS for FLLs' characterization was assessed in terms of lesion status and specific lesion type. The sensitivity ( $\mathrm{Se}$ ) was calculated as true positive cases divided by the total number of cases in which the disease was present; the specificity (Sp) was calculated as true negative cases divided by the total number of cases in which the disease was absent; the positive predictive value (PPV) was calculated as true positive cases divided by all CEUS positive cases; the negative predictive value (NPV) was calculated as true negative cases divided by all CEUS negative cases and accuracy was calculated as the sum of true positive and true negative cases divided by the total number of cases. We included in the statistical analysis all cases reported, while the inconclusive CEUS cases were considered as wrongly diagnosed.

\section{Results}

During February 2011 - May 2015, 1153 CEUS examinations were performed for the evaluation of de novo FLL. Out of the 1153 de novo FLL, 238 cases were di- 
agnosed as hemangiomas by CEUS (typical enhancing pattern). Contrast CT/MRI and biopsy diagnosed additional 24 hemangiomas (17 in inconclusive CEUS cases, 4 in cases diagnosed as hepatocellular carcinomas by CEUS, 2 in cases diagnosed as focal fatty infiltration by CEUS and 1 in a case diagnosed as dysplastic nodule by CEUS). From the 238 cases diagnosed as hemangiomas by CEUS, in 11 the final diagnosis was different: in 5 cases hepatocellular carcinoma, in 1 case adenoma, in 1 case focal nodular hyperplasia, 1 case dysplastic nodule, 1 case inflammatory lesion, 2 cases inconclusive.

Considering contrast CT/MRI as reference method, CEUS had $90.4 \%$ sensitivity, $98.8 \%$ specificity, $95.4 \%$ positive predictive value, $97.4 \%$ negative predictive value, resulting in $96.9 \%$ diagnostic accuracy for the diagnosis of hemangiomas.

\section{Discussions}

As showed above, hemangiomas are the most frequent benign tumors of the liver and thus frequently discovered in clinical practice, either during a "routine" US examination performed for unrelated pathology, or during the initial evaluation or follow up in patients with chronic hepatitis or oncologic diseases. Even if the B-mode US appearance of hemangioma is "typical" in most cases, the same US aspect can be found in patients with hepatocellular carcinoma (HCC) or liver metastases. Thus, a FLL suspected to be a hemangioma based on "typical" B-mode US aspect, found in a patient with liver cirrhosis (a risk factor for HCC) or with a malignant tumor (regardless of the primary location) should undergo a second-line imaging technique to confirm the diagnosis. In patients with no such history it is acceptable to repeat B-mode US after three or six months to document the FLL's stable aspect. Also, patients with known hepatic hemangioma who subsequently develop liver disease or extrahepatic malignancy should be further evaluated by second-line imaging methods [25].

Until recently, contrast enhanced CT and MRI were considered the best second-line imaging methods to confirm an US diagnosis of hemangioma. MRI is considered to be the best method since it is non-invasive and highly accurate, with approximately $90 \%$ sensitivity and $91-99 \%$ specificity [26]. The typical MRI appearance in hemangioma is a smooth, well-demarcated homogeneous mass that has low signal intensity on T1-weighted images and is hyperintense on T2-weighted images [26]. The presence of intratumoral fibrosis results in areas of low intensity on T2-weighted images. Administration of gadolinium results in early peripheral discontinuous nodular or globular enhancement on arterial phase with progressive centripetal enhancement or "filling-in" on delayed scans similar to that seen on CT scanning [25].

Technetium-99m pertechnetate-labeled red blood cell pool study (99mTc-RBC pool studies) is another technique useful for the diagnosis of hemangioma. Initial hypoperfusion is observed during arterial flow, followed by gradual increase of tracer peaking 30 to 50 minutes after the injection. The isotope is retained within the lesion on delayed images. Sensitivity of $99 \mathrm{mTc}-\mathrm{RBC}$ pool studies for FLL larger than $2 \mathrm{~cm}$ varies from $69-92 \%$, with almost $100 \%$ specificity [27].

Considering that contrast enhanced imaging methods are highly accurate for the diagnosis of hemangioma, biopsy is performed only in cases in which imaging techniques are not conclusive. According the American College of Radiology guidelines, liver biopsy should be performed only in indeterminate (after contrast $\mathrm{CT} /$ MRI) liver lesions $>1 \mathrm{~cm}$ in diameter, in patients with a history of malignancy or of chronic liver disease [28]. Even if complications, especially bleeding, can occur following hemangioma biopsy, once the typical vascular hemangiomas are excluded, there is a surprisingly low risk of significant complications in atypical hemangiomas. The reason may be that thrombosis or fibrosis in the lesion, which makes the imaging appearance atypical, also reduces the potential for bleeding complications [29]. Ultrasound-guidance, normal bleeding parameters, suitable trajectory planning via a small cuff of normal liver may all contribute to the low complication rate [30].

Contrast CT and MRI are expensive and available only in specialized centers. CT is also an irradiating procedure, while contrast agents used for CT can trigger or worsen renal failure. SonoVue microbubbles are strictly intravascular (as opposed to contrast agents in CT and MRI that diffuse into the interstitium) and include hexafluoride sulfur, a biologically inert gas that is cleared through exhalation (not through the kidney as CT or MRI contrast agents), therefore it is not contraindicated in patients with renal failure [6-8].

Published data showed very good results regarding the diagnostic performance of CEUS for hemangioma. In the German multicentre study that included 1,349 patients with FLL in which CEUS was compared with biopsy in more than $75 \%$ of lesions, or with spiral contrast CT or contrast MRI, CEUS correctly diagnosed $82.2 \%$ of hemangiomas [2]. In the multicentre French study (STIC), which included 1034 FLL and in which CEUS was also compared to contrast spiral CT, contrast MRI or liver biopsy, considered to be the "gold standard", CEUS had $85.4 \%$ sensitivity and $93.7 \%$ specificity for the diagnosis of hemangioma [3]. 
In another multinational study on 134 patients in which CEUS was compared to contrast-enhanced CT and/or dynamic contrast-enhanced MRI, CEUS had $100 \%$ sensitivity and $87 \%$ specificity for the identification of focal nodular hyperplasia (FNH) and hemangioma, resulting in an accuracy of $94.5 \%$ [31].

In a recently published study, on 83 lesions, the accuracies of CEUS and contrast enhanced MRI in diagnosing hemangiomas were compared considering histology as the reference method. There were no significant differences in the diagnostic value between CEUS and MRI in terms of sensitivity $(88.0 \%$ vs. $92.8 \%)$, specificity ( $99.0 \%$ vs. $99.4 \%$ ), accuracy ( $97.3 \%$ vs. $98.4 \%$ ), positive predictive value (93.6\% vs. $96.3 \%)$, and negative predictive value $(98.0 \%$ vs. $98.8 \%)(\mathrm{p}>0.05$, all) [32]

Similar to the studies presented above, in our study CEUS was highly accurate $(96.9 \%)$ for the diagnosis of hemangioma (90.4\% sensitivity, $98.8 \%$ specificity, $95.4 \%$ positive predictive value, $97.4 \%$ negative predictive value). In our study contrast CT/MRI and biopsy diagnosed additional 24 hemangiomas: mostly (17 hemangiomas) in inconclusive CEUS cases, but also in cases misdiagnosed by CEUS (4 HCC, 2 focal fatty infiltration, and 1 dysplastic nodule). Also, from the 238 cases diagnosed as hemangiomas by CEUS, in 11 the final diagnosis was different: 5 HCCs, 1 adenoma, 1 focal nodular hyperplasia, 1 dysplastic nodule, 1 case inflammatory lesion, 2 cases inconclusive by contrast CT/MRI.

Which are the causes for misdiagnosis? In a paper that analyzed unclear FLL after CEUS in the German multicentre study, 31 benign lesions, from which 9 biopsy proven hemangiomas, were classified as malignant by CEUS and 86 lesions were unclear after CEUS (67 benign lesions iso- or hypoenhancing during the late phases, from which 20 hemangiomas) [33]. The authors suggested that hypo- or isoenhancement of hemangiomas in the late phases of CEUS could be due to the continuous insonation of the lesion which leads to bubble destruction, especially in the near field (fig 2). In our study, from the 24 hemangiomas additionally diagnosed by contrast CT/MRI, 11 were in inconclusive cases by CEUS (declared inconclusive due to hypoenhancement in the late phase even if centripetal, nodular fill in was observed in the arterial phase).

Apart from inconclusive cases by CEUS later diagnosed as hemangiomas by contrast enhanced CT/MRI or biopsy, the highest number of misdiagnosed cases were HCCs: 4 cases diagnosed by CEUS as HCCs and proven to be hemangiomas (all in patients with chronic hepatitis and cirrhosis) and 5 cases diagnosed by CEUS to be hemangiomas and proven to be HCCs by second-line imaging methods or biopsy ( 3 in patients with chronic

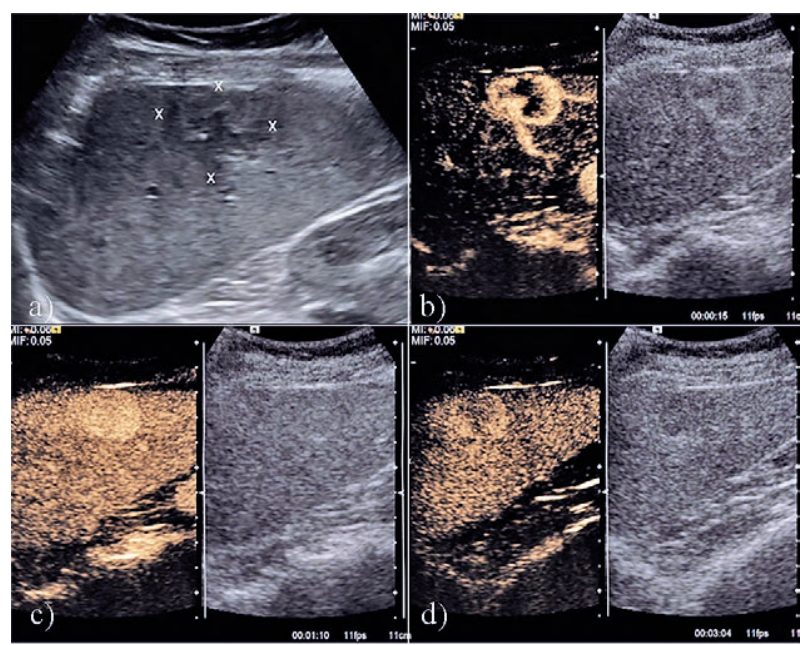

Fig 2. a) Isoechoic, inhomogeneous nodule, situated subcapsular in segment VI of the liver; b) CEUS - arterial phase: nodular, peripheral enhancement of the nodule can be observed; c) CEUS - portal phase: the nodule is completely hyperenhanced; d) CEUS - late phase: the nodule is hyperenhancing in the periphery, but the central area is hypoenhancing.

hepatitis and cirrhosis). HCCs are lesions that show arterial hyperenhancement and wash-out in the portal and late phases [6-8]. The arterial enhancement in HCC is usually homogeneous, but it can also be heterogeneous, due to fatty degeneration or intratumoral necrosis [34,35], while in well-differentiated HCCs, wash-out can occur only in late phases $[8,36]$. Considering all of the above, we can speculate that the 5 HCCs misdiagnosed by CEUS as hemangiomas, were well-differentiated and thus the wash-out was not visible during the 5 minutes of evaluation. Regarding the 4 hemangiomas misdiagnosed by CEUS as HCCs, we can speculate that a pseudo washout was observed due to hyperinsonation as showed in the German study $[33,34]$, while the arterial inhomogeneous hyperenhancement observed in these cases can be explained by arterio-venous and/or portal venous shunts, known to be present in $10 \%$ of hemangiomas [37]. Also, rapidly filling hemangiomas, usually smaller than $3 \mathrm{~cm}$ can be encountered in patients with cirrhosis in up to 16$18 \%$ of cases $[30,38,39]$

Previous studies demonstrated that CEUS accuracy for the characterization of FLL is comparable to that of contrast CT [40] and contrast MRI [41], and also that the use of CEUS as a first line imaging method when faced with a new FLL is cost-efficient $[42,43]$. Also the results of our study and of those mentioned above demonstrate very good accuracy for the diagnosis of hemangioma, so we can safely suggest to use CEUS a first-line imaging technique when faced with a suspected hemangioma based on standard B-mode US, even in high risk patients 
in which clinical and serologic data should also be taken into consideration.

A limitation of our study is that we didn't include consecutive patients, but only those who met the inclusion criteria (having a second-line reference method: contrast CT/MRI or liver biopsy).

\section{Conclusion}

CEUS is a sensitive (90.4\%) and very specific $(98.8 \%)$ method for the diagnosis of hemangiomas, with an overall $96.9 \%$ diagnostic accuracy and should be considered as a first-line imaging technique when faced with a suspected hemangioma based on standard B-mode US, even in high risk patients.

\section{Conflict of interest: none}

\section{Acknowledgements:}

The research published in this paper was made with support from the grant awarded by the "Victor Babeş" University of Medicine and Pharmacy Timisoara, in Programul III - C2 - PCFI - 2015/2016.

The authors wish to acknowledge the help provided by the following colleagues: Dorina Pestroiu Calescu ${ }^{4}$, Dana Nedelcu ${ }^{5}$, Ciprian Brisc ${ }^{6}$, Lucian Ciobâca ${ }^{7}$, Liana Gheorghe ${ }^{8}$, Mihai Socaciu ${ }^{3}$, Alina Martie ${ }^{1}$, Simona Ioaniţescu ${ }^{9}$, Attila Tamas ${ }^{10}$, Costin Theodor Streba ${ }^{2}$, Mihaela Iordache ${ }^{7}$, Iulia Simionov ${ }^{8}$, Mariana Jinga ${ }^{7}$, Adrian Anghel $^{7}$, Cristina Cijevschi Prelipcean ${ }^{11}$, Cătălina Mihai ${ }^{11}$, Silviu Marcel Stanciu ${ }^{7}$, Daniel Stoicescu ${ }^{7}$, Eugen Dumitru $^{12}$, Corina Pietrăreanu ${ }^{8}$, Daniela Bartoş ${ }^{13}$, Roberta Mânzat Saplacan ${ }^{14}$, Iuliana Pârvulescu ${ }^{8}$, Roxana Vădan ${ }^{8}$, Gabriela Smira ${ }^{8}$, Liliana Tuţă ${ }^{12}$,

${ }^{1}$ Department of Gastroenterology and Hepatology, "Victor Babeş" University of Medicine and Pharmacy Timişoara, ${ }^{2}$ Research Centre in Gastroenterology and Hepatology, University of Medicine and Pharmacy Craiova, ${ }^{3}$ Regional Institute of Gastroenterology and Hepatology "Prof. Dr. Octavian Fodor", "Iuliu Hațieganu" University of Medicine and Pharmacy Cluj Napoca, ${ }^{4}$ Department of Gastroenterology, Emergency Hospital Floreasca, Bucharest, ${ }^{5}$ Delta Promedical Hospital, Bucharest, ${ }^{6}$ Department of Gastroenterology, University of Oradea, ${ }^{7} 1^{\text {st }}$ Medical Department, Central Military Emergency Hospital, "Dr. Carol Davila" University, Bucharest, ${ }^{8} \mathrm{III}^{\text {rd }}$ Department of Gastroenterology and Hepatology, Clinical Institute Fundeni, Bucharest, ${ }^{9} \mathrm{Center}$ of Internal Medicine, Clinical Institute Fundeni, Bucharest, ${ }^{10}$ Department of Anatomy and Embriology, "Iuliu Haţieganu" University of Medicine and Pharmacy Cluj Napoca, ${ }^{11}$ Institute of Gastroenterology and Hepatology,
"Grigore T. Popa" University of Medicine and Pharmacy Iaşi, ${ }^{12}$ Department of Gastroenterology, University of Constanţa, ${ }^{13}$ Department of Internal Medicine and Gastroenterology, Emergency Hospital Floreasca, Bucharest, ${ }^{14}{ }^{\text {st }}$, Medical Clinic, "Iuliu Haţieganu" University of Medicine and Pharmacy, Cluj Napoca, Romania

\section{References}

1. Rumack CM, Wilson SR, Charboneau JW, et al. Diagnostic ultrasound. 3 rd ed. Elsevier Mosby. St. Louis, 2005: 121293.

2. Strobel D, Seitz K, Blank W, et al. Contrast-enhanced ultrasound for the characterization of focal liver lesions-diagnostic accuracy in clinical practice (DEGUM multicenter trial). Ultraschall Med 2008; 29: 499-505.

3. Tranquart F, Le Gouge A, Correas JM, et al. Role of contrast-enhanced ultrasound in the blinded assessment of focal lesions in comparison with MDCT and CEMRI: Results from a multicentre clinical trial. EJC Supplements 2008; 6: 9-15.

4. Xie L, Guang Y, Ding H, Cai A, Huang Y. Diagnostic value of contrast-enhanced ultrasound, computed tomography and magnetic resonance imaging for focal liver lesions: a meta-analysis.Ultrasound Med Biol 2011; 37: 854-861.

5. Friedrich-Rust M, Klopffleisch T, Nierhoff J, et al. Contrast Enhanced Ultrasound for the differentiation of benign and malignant focal liver lesions: a meta-analysis. Liver Int 2013; 33: 739-755.

6. Albrecht T, Blomley M, Bolondi L, et al; EFSUMB Study Group. Guidelines for the use of contrast agents in ultrasound. January 2004. Ultraschall Med 2004; 25: 249-256.

7. Claudon M, Cosgrove D, Albrecht T, et al. Guidelines and good clinical practice recommendations for contrast enhanced ultrasound (CEUS) - update 2008. Ultraschall Med 2008; 29: 28-44.

8. Claudon M, Dietrich CF, Choi BI, et al. Guidelines and good clinical practice recommendations for Contrast Enhanced Ultrasound (CEUS) in the liver - update 2012: A WFUMB-EFSUMB initiative in cooperation with representatives of AFSUMB, AIUM, ASUM, FLAUS and ICUS. Ultrasound Med Biol 2013; 39: 187-210.

9. Moody AR, Wilson SR. Atypical hepatic hemangioma: a suggestive sonographic morphology. Radiology 1993; 188: 413-417.

10. Semelka RC, Sofka CM. Hepatic hemangiomas. Magn Reson Imaging Clin N Am 1997; 5: 241-253.

11. Leifer DM, Middleton WD, Teefey SA, Menias CO, Leahy JR. Follow-up of patients at low risk for hepatic malignancy with a characteristic hemangioma at US. Radiology 2000; 214: 167-172.

12. Tait N, Richardson AJ, Muguti G, Little JM. Hepatic cavernous haemangioma: a 10 year review. Aust N Z J Surg 1992; 62: 521-524.

13. Grieco MB, Miscall BG. Giant hemangiomas of the liver. Surg Gynecol Obstet 1978; 147: 783-787. 
14. Saegusa T, Ito K, Oba N, et al. Enlargement of multiple cavernous hemangioma of the liver in association with pregnancy. Intern Med 1995; 34: 207-211.

15. Conter RL, Longmire WP Jr. Recurrent hepatic hemangiomas. Possible association with estrogen therapy. Ann Surg 1988; 207: 115-119.

16. Massironi S, Branchi F, Rossi RE, et al. Hepatic hemangioma in celiac patients: data from a large consecutive series. Gastroenterol Res Pract 2015; 2015: 749235.

17. Berzigotti A, Frigato M, Manfredini E, et al. Liver hemangioma and vascular liver diseases in patients with systemic lupus erythematosus. World J Gastroenterol 2011; 17: 4503-4508.

18. Ishak KG, Rabin L. Benign tumors of the liver. Med Clin North Am 1975; 59: 995-1013.

19. Goodman Z. Benign Tumors of the Liver. In: Okuda K, Iskah E (Eds). Neoplasms of the liver. Springer-Verlag, Tokyo 1987: 105-

20. Pateron D, Babany G, Belghiti J, et al. Giant hemangioma of the liver with pain, fever, and abnormal liver tests. Report of two cases. Dig Dis Sci 1991; 36: 524-527.

21. McArdle CR. Ultrasonic appearances of a hepatic hemangioma. J Clin Ultrasound 1978; 6: 124.

22. Bree RL, Schwab RE, Glazer GM, Fink-Bennett D. The varied appearances of hepatic cavernous hemangiomas with US, CT, MR imaging and scintigraphy. Radiographics 1987; 7: 1153-1175.

23. Moody AR, Wilson SR. Atypical hemangioma: a suggestive US morphology. Radiology 1993; 188: 413-417.

24. Perkins AB, Imam K, Smith WJ, Cronan JJ. Color and power Doppler sonography of liver hemangiomas: a dream unfulfilled? J Clin Ultrasound 2000; 28: 159-165.

25. Curry MP, Chopra S. Hepatic hemangioma. On-line paper. Available at: http://www.uptodate.com/online/content/topic.do?topicKey=hep_dis/19680\&selectedTitle $=1 \sim 150 \&$ so urce $=$ search_result. Accessed 05.09.2015.

26. Lee MG, Baker ME, Sostman HD, et al. The diagnostic accuracy/efficacy of MRI in differentiating hepatic hemangiomas from metastatic colorectal/breast carcinoma: a multiple reader ROC analysis using a jackknife technique. J Comput Assist Tomogr 1996; 20: 905-913.

27. Farlow DC, Chapman PR, Gruenewald SM, Antico VF, Farrell GC, Little JM. Investigation of focal hepatic lesions: is tomographic red blood cell imaging useful? World J Surg 1990; 14: 463-467.

28. Nelson RC, Kamel IR, Baker ME, et al. Expert Panel on Gastrointestinal Imaging. ACR Appropriateness Criteria ${ }^{\circledR}$ liver lesion -- initial characterization. Reston (VA): American College of Radiology (ACR); 2014. On-line paper. Available at: http://www.guideline.gov/content. aspx?id=48283. Accessed 05.09.2015.

29. Alamdaran A, Esmailzadeh A, Noroozi HR, Mohajeri HR, Yazdani AA. Ultrasound-guided Core Needle Biopsy of Liver Hemangiomas: Three-Year Experience. Iran J Radiol 2007; 4: 255-258.

30. Vilgrain V, Boulos L, Vullierme MP, Denys A, Terris B, Menu Y. Imaging of atypical hemangiomas of the liver with pathologic correlation. Radiographics 2000; 20: 379397.

31. Trillaud H, Bruel JM, Valette PJ, et al. Characterization of focal liver lesions with SonoVue ${ }^{\circledR}$ - enhanced sonography: International multicenter-study in comparison to $\mathrm{CT}$ and MRI. World J Gastroenterol 2009; 15: 3748-3756.

32. Fang L, Zhu Z, Huang B, et al. A comparative study of contrast enhanced ultrasound and contrast enhanced magnetic resonance imaging for the detection and characterization of hepatic hemangiomas. Biosci Trends 2015; 9: 104-110.

33. Bernatik T, Seitz K, Blank W, Schuler A, Dietrich CF, Strobel D. Unclear focal liver lesions in contrast-enhanced ultrasonography-lessons to be learned from the DEGUM multicenter study for the characterization of liver tumors Ultraschall Med 2010; 31: 577-581.

34. Giannetti A, Franci L, Grechi C, Giangregorio F. Contrastenhanced sonography in the diagnosis of hepatic hemangiomas: atypical appearance due to the washout of microbubbles. J Clin Ultrasound 2013; 41: 361-365.

35. Zhu AX, Chabner BA, Tanabe KK. New trends and novel treatment for hepatocellular carcinoma: a global perspective. Oncologist 2010: 15 Suppl 4: 1-4-

36. Guo LH, Xu HX. Contrast-Enhanced Ultrasound in the Diagnosis of Hepatocellular Carcinoma and Intrahepatic Cholangiocarcinoma: Controversy over the ASSLD Guideline. Biomed Res Int 2015; 2015: 349172.

37. Dietrich CF, Mertens JC, Braden B, Schuessler G, Ott M, Ignee A. Contrast-enhanced ultrasound of histologically proven liver hemangiomas. Hepatology 2007; 45: 1139 1145 .

38. Brancatelli G, Federle MP, Blachar A, Grazioli L. Hemangioma in the cirrhotic liver: diagnosis and natural history. Radiology 2011; 219: 69-74.

39. Quaia E, Bartolotta TV, Midiri M, Cernic S, Belgrano M, Cova M. Analysis of different contrast enhancement patterns after microbubble-based contrast agent injection in liver hemangiomas with atypical appearance on baseline scan. Abdom Imaging 2006; 31: 59-64.

40. Seitz K, Strobel D, Bernatik T, et al. Contrast-Enhanced Ultrasound (CEUS) for the characterization of focal liver lesions - prospective comparison in clinical practice: CEUS vs. CT (DEGUM multicenter trial). Ultraschall Med 2009; 30: 383-389.

41. Seitz K, Bernatik T, Strobel D, et al. Contrast-Enhanced Ultrasound (CEUS) for the Characterization of Focal Liver Lesions in Clinical Practice (DEGUM Multicenter Trial): CEUS vs. MRI - a Prospective Comparison in 269 Patients. Ultraschall Med 2010; 31: 492-499.

42. Giesel FL, Delorme S, Sibbel R, Kauczor HU, Krix M. Contrast-enhanced ultrasound for the characterization of incidental liver lesions - an economical evaluation in comparison with multi-phase computed tomography. Ultraschall Med 2009; 30: 259-268.

43. Sirli R, Sporea I, Martie A, Popescu A, Dănilă M. Contrast enhanced ultrasound in focal liver lesions--a cost efficiency study. Med Ultrason 2010; 12: 280-285. 